ISSN 1991-8631

Original Paper

http://indexmedicus.afro.who.int

\title{
Caractéristiques floristiques, diversité et structure de la végétation ligneuse dans le Centre-Sud du Niger : cas du complexe des forêts classées de Dan kada Dodo-Dan Gado
}

\author{
Hamidou ABDOURHAMANE ${ }^{1,2^{*}}$, Boubé MOROU ${ }^{1}$, Habou RABIOU ${ }^{1}$ et \\ Ali MAHAMANE ${ }^{1}$ \\ ${ }^{1}$ Université de Maradi, BP 465 Maradi, Niger. \\ 2 Projet PRACC, BP 223 Niamey, Niger. Tel (00227) 20752337 \\ *Auteur correspondant ; E- mail: hamidyoro@yahoo.fr; BP 223 Niamey ; Tel (00227) 98889998
}

\section{RESUME}

Les forêts classées de Dan kada Dodo et Dan Gado, constituant le complexe forestier d'une superficie 11630 ha, sont situées dans la région de Maradi au centre-sud du Niger. La présente étude vise à caractériser la végétation ligneuse de ce complexe forestier sur le plan de sa composition floristique et de sa structure. Les données ont été collectées au moyen des relevés floristiques, de mesure du diamètre à 1,30 m pour les arbres et par le comptage des individus à diamètre $<5 \mathrm{~cm}$ dans 288 placettes de 20 x $20 \mathrm{~m}$. Les indices de diversité ont été calculés sur la base des fréquences spécifiques. L'analyse a mis en évidence une faible richesse floristique avec 31 espèces ligneuses appartenant à 15 familles dont les plus dominantes sont les Mimosaceae $(24,24 \%)$ et Combretaceae $(15,15 \%)$. Les microphanérophytes sont plus abondants $(94,24 \%)$, marquant le caractère arbustif de la végétation du complexe forestier. La chorologie de la flore est caractérisée par des espèces à distribution Soudano-Zambézienne (59,5\%) suivies des Soudaniennes (31,8\%). La faible densité des ligneux $(425,19 \pm 08$ individus/ha) serait due entre autres aux conditions climatiques précaires et à la pression des populations riveraines. La structure diamétrique s'ajuste à la distribution de Weibull et montre une prédominance des individus jeunes.

(C) 2013 International Formulae Group. All rights reserved.

Mots clés: Composition floristique, spectre biologique, diversité, densité, structure.

\section{INTRODUCTION}

La dégradation des écosystèmes forestiers constitue une des causes de réduction de la biodiversité dans le monde (Oszwald, 2005 ; N'da et al., 2008). Si, au niveau global, les pertes forestières pour la période 2005-2010 étaient de 0,14\% par an, l'Afrique de l'ouest se distingue par un taux de déboisement estimé à $0,46 \%$ (FAO, 2010). La zone sahélienne du Comité Inter-Etats de Lutte contre la Sécheresse dans le Sahel (CILSS), pourtant caractérisée par des ressources forestières limitées et plus vulnérables, connait un recul annuel de 1,07\% des formations forestières au cours de la période 2005-2010 (FAO, 2010). La péjoration climatique associée à d'autres facteurs anthropiques comme les défrichements agricoles, le surpâturage, les 
prélèvements de bois d'œuvre et d'artisanat, de bois de service et d'énergie, les feux de brousse, ont été à l'origine de dégradations parfois profondes de ces formations forestières y compris celles $\mathrm{du}$ domaine protégé (Boulain, 2004; Sambou, 2004; Ozer et Ozer, 2005; Vroh Bi Tra et al., 2010; Gonzalez et al., 2012). Plusieurs auteurs dont Ganaba (2008) et Paré (2008) au Burkina Faso; Larwanou et Saadou (2005) au Niger, ont signalé la régression de la densité et de la qualité des espèces ligneuses dans la zone sahélienne à telle enseigne que beaucoup d'espèces deviennent rares ou s'éteignent dans leur aire de distribution.

L'importance des aires protégées dans la conservation de la biodiversité est actuellement largement reconnue (Mahamane, 2005 ; Paré, 2008; Ouédraogo, 2009). En effet, elles servent de refuges à de nombreuses espèces et constituent pour les populations locales un patrimoine exceptionnel du fait des biens et des services qu'elles leur offrent. Par ailleurs, la végétation ligneuse joue un rôle important dans le maintien de l'équilibre écologique, dans un milieu sahélien qui fait face à l'extension du désert (Breman et Kessler, 1995; Hountondji, 2008).

Cette prise de conscience face à la préservation de la biodiversité s'est traduite par la mise en place des dispositions réglementaires pour protéger les terres forestières. Cependant, force est de constater que même si le classement est une mesure de conservation et de protection qui consiste à doter une réserve d'un régime spécial, restrictif, concernant son exploitation et l'exercice des droits d'usages coutumiers, ces domaines protégés subissent encore des empiétements agricoles. D'où la nécessité de mettre en place une approche d'utilisation durable des ressources naturelles et de préservation de la biodiversité (Mahamane, 2005). Mais, l'insuffisance de données scientifiques constitue une contrainte qui limite la mise en œuvre des pratiques d'aménagement et de gestion durable de la biodiversité. La présente étude rentre dans ce cadre et a pour objectif de caractériser la végétation ligneuse du complexe des forêts classées de Dan Kada Dodo-Dan Gado à travers l'analyse de sa composition floristique, de la structure de ses communautés végétales et des paramètres écologiques.

\section{MATERIEL ET METHODES Site d'étude}

Les forêts classées de Dan Kada Dodo et de Dan Gado se situent entre les latitudes $13^{\circ} 27^{\prime}$ à $13^{\circ} 35^{\prime}$ Nord et les longitudes $07^{\circ}$ $34^{\prime}$ à $07^{\circ} 43^{\prime}$ Est dans la région de Maradi au centre-sud du Niger (Figure 1). D'une superficie totale de 11630 ha, ces deux forêts constituant le complexe, se caractérisent par un relief peu accidenté, marqué par un plateau muni de quelques buttes de sable avec des surfaces quasi-planes et de petites dépressions (Matsallabi, 2008). Du point de vue climatique, deux principales saisons sont observées au cours de l'année, l'une sèche de sept mois environ et l'autre humide qui dure de mai à septembre. La moyenne pluviométrique durant les dix dernières années est de 483,74 $\pm 124,36 \mathrm{~mm}$. Trois types de sols y sont distingués: les sols dunaires d'apports éoliens de structure sablonneuse, les sols ferrugineux de structure limoneuse des plateaux et les sols hydromorphes à texture argilo-sableuse localisés dans les zones de dépression (Matsallabi, 2008). Sur le plan phytogéographique, le complexe des forêts classées de Dan kada Dodo-Dan Gado (FDKDG) se trouve dans le Compartiment Sud-Sahélien (Saadou, 1990). La végétation est une steppe en dégradation. La population dans la zone est estimée à 386197 habitants en 2011 soit une densité de $137 \mathrm{hbts} / \mathrm{km}^{2}$. L'agriculture et l'élevage constituent les principales activités pratiquées de façon extensive. 


\section{Méthodes \\ Echantillonnage}

La méthode d'échantillonnage stratifié suivant les types d'utilisation des terres a été adoptée. Elle a consisté à faire un échantillonnage à l'intérieur de 7 principales unités d'occupation des terres identifiées suite à la cartographie du complexe forestier (Figure 1). Cette carte d'occupation des terres de 2011 a subi un maillage. Pour chaque unité d'occupation des terres, un tirage aléatoire des mailles caractéristiques de $250 \mathrm{~m}$ x $250 \mathrm{~m}$ a été effectué grâce à la fonction «Alea» du programme Excel. Une maille est dite caractéristique si elle est entièrement incluse dans l'unité d'occupation des terres. A l'intérieur de chaque maille échantillonnée, 8 placettes d'inventaire de $20 \mathrm{~m}$ x $20 \mathrm{~m}$ (soit $400 \mathrm{~m}^{2}$ ) sont installées suivant les huit directions ( $\mathrm{E}, \mathrm{O}, \mathrm{N}, \mathrm{S}, \mathrm{NE}, \mathrm{SE}, \mathrm{NO}, \mathrm{SO})$ à des distances aléatoires dans une zone circulaire de 115 mètres de rayon ayant pour centre l'intersection des deux médianes de la maille. Cette distance permet d'éviter un débordement de la placette par rapport à la limite de la maille (Sambou, 2004). Au total, 36 mailles totalisant 288 placettes ont été inventoriées représentant 11,52 hectares (soit un taux d'échantillonnage de $0,01 \%$ ).

\section{Collecte des données}

La collecte de données floristique et dendrométrique a été effectuée dans les placettes de 20 × $20 \mathrm{~m}$ au mois de septembre 2011 et 2012. La nomenclature des espèces adoptée est celle de Lebrun et Stork (1997). Les mesures dendrométriques ont porté sur la hauteur totale, le diamètre du houppier dans les deux directions perpendiculaires, le diamètre à $1,30 \mathrm{~m}$ du sol pour les individus à $\mathrm{dbh} \geq 5 \mathrm{~cm}$ et à $20 \mathrm{~m} \mathrm{du}$ sol pour les arbustes (Sambou, 2004; Ouédraogo, 2009). Les individus dont le diamètre est inférieur à $5 \mathrm{~cm}$ sont considérés comme faisant partie de la régénération (Mahamane et Saadou, 2008). Ces individus ont été comptés et regroupés en trois classes: Classe 1 pour les individus dont la hauteur est moins de $1,30 \mathrm{~m}$; Classe 2 pour ceux dont la hauteur supérieure à $1,30 \mathrm{~m}$ et Classe 3 pour ceux issus du semis naturel.

\section{Analyse des données}

Les données structurales combinées aux données floristiques ont permis de calculer l'indice de valeur d'importance (IVI) ou Importance Value Index (IVI) de Curtis et Macintosh (1951) cités par Traoré (1997). Cet indice s'exprime selon la formule suivante:

IVI $=$ Densité relative + Dominance relative + Fréquence relative (pour l'espèce).

Où la dominance relative d'une espèce est le quotient de son aire basale avec l'aire basale totale de toutes les espèces; la densité relative d'une espèce est le rapport de sa densité absolue au total des densités absolues de toutes les espèces multiplié par cent et la fréquence relative d'une espèce est le rapport de sa fréquence spécifique par le total des fréquences spécifiques de toutes les espèces multiplié par cent.

Les types biologiques ont été déterminés en se référant aux travaux de Raunkiaer (1934), repris et aménagés par diverses études de la végétation des régions tropicales (Guinko, 1984 ; Saadou, 1990). Pour la strate ligneuse, il s'agit essentiellement de phanérophytes qui ont été subdivisés en Nanophanérophytes ( $\mathrm{NnPh}$ ), arbustes de 0,5 à $2 \mathrm{~m}$ de hauteur; Microphanérophytes $(\mathrm{McPh})$, arbustes de 2 à $8 \mathrm{~m}$ de hauteur; Mésophanérophytes $(\mathrm{MsPh})$, arbres moyens de 8 à $30 \mathrm{~m}$ hauteur et Mégaphanérophytes (MPh), grands arbres de plus de $30 \mathrm{~m}$ de hauteur.

Les éléments phytogéographiques ont été définis en se référant à ceux définis par White (1986) utilisés par plusieurs auteurs pour les phytochories africaines (Sinsin, 1993; Mahamane, 2005). Il s'agit des espèces à large distribution comme les espèces afroaméricaines (AA), pantropicales (Pan), Paléotropicales $(\mathrm{Pal})$; espèces à distribution continentale regroupant les espèces SoudanoGuinéennes (SG), Afro-Tropicales (AT), Afro-Malgaches (AM), Pluri-Régionales 
Africaines (PRA), Soudano-Zambéziennes (SZ) et Guinéo-Congolaises (GC) et des espèces de l'élément-base Soudanien (S) distribuées dans le Centre Régional d'Endémisme (CRE) soudanien.

L'analyse de la diversité spécifique a été faite à l'aide des indices de diversité de Shannon-Weaver et l'indice d'équitabilité de Pielou et la richesse spécifique (nombre d'espèces). Ces indices s'expriment par :

- $\quad$ l'indice de Shannon $(\mathrm{H}): \mathrm{H}=-\Sigma$ pi $\log _{2}$ pi. Avec pi $=$ ni/N, abondance relative de l'espèce $\mathrm{i}$ dans l'échantillon total auquel appartient l'espèce, d'où $\mathrm{N}=$ effectif total des individus, $\mathrm{ni}=$ effectif des individus de l'espèce $i$. L'indice $\mathrm{H}$ est exprimé en bit et est utilisé pour apprécier l'hétérogénéité et la diversité d'un biotope.

- $\quad$ l'équitabilité de Pielou $\mathrm{E}=\mathrm{H} / \log _{2} \mathrm{~S}$, correspond au rapport entre la diversité observée de Shannon $(\mathrm{H})$ et la diversité maximale (Hmax) possible étant donné le nombre d'espèces $\mathrm{S}$. Elle est très utile pour comparer les dominances potentielles entre stations.

La densité moyenne des ligneux a été calculée pour l'ensemble du complexe forestier et pour chacune des unités d'occupation des terres identifiées.

Le recouvrement $(\mathrm{R})$ est calculé par la formule suivante : $\mathrm{R}(\%)=(\mathrm{Sh} / \mathrm{Ss}) \times 100$ avec $\mathrm{R}=$ le taux de recouvrement exprimé en (\%); $\mathrm{Sh}=$ Surface du houppier $=\pi(\mathrm{Dmh} / 2)^{2} ; \mathrm{Ss}=$ Surface d'inventaire et $\mathrm{Dmh}=$ diamètre moyen du houppier.

La surface terrière des arbres a été calculée pour l'ensemble du complexe forestier, pour chaque unité d'occupation des terres suivant la formule (Mahamane et Saadou, 2008):

$\mathrm{G}\left(\mathrm{m}^{2} / \mathrm{ha}\right)=\frac{\pi}{40000 \mathrm{~s}} \sum_{\mathrm{i}}^{\mathrm{n}} \mathrm{d}_{\mathrm{i}}^{2}$

Où $\mathrm{d}_{\mathrm{i}}$ : diamètre (en mètre) de l'arbre $\mathrm{i}$ de la placette; $\mathrm{s}$ : aire surface de la placette en $\mathrm{m}^{2}$ et л $=3,1416$.
L'analyse de la régénération a porté sur le calcul de la densité des tiges et le taux de régénération exprimé en pourcentage (\%) correspondant à la proportion de jeunes tiges par rapport à la densité de tiges pour chacune des 3 classes de régénération adoptées.

L'analyse de la structure démographique des ligneux s'est effectuée à travers des histogrammes de distribution de fréquences relatives calculées par classe de diamètre. Pour ce faire, 15 classes d'amplitude $5 \mathrm{~cm}$ ont été définies. En outre, pour mieux caractériser la variabilité des formes des structures observées et de rendre possible les comparaisons entre structures, un ajustement à la distribution théorique de Weibull basée sur la méthode du maximum de vraisemblance a été appliquée (Husch et al., 2003 cité par Morou, 2010) avec le logiciel Minitab 14.1. Elle se fonde sur la fonction de densité de probabilité de la distribution de Weibull $F$ définie par:

$$
\mathrm{F}(\mathrm{x})=\mathrm{c} / \mathrm{b}((\mathrm{x}-\mathrm{a}) / \mathrm{b})^{\mathrm{c}-1} \exp \left(-((\mathrm{x}-\mathrm{a}) / \mathrm{b})^{\mathrm{c}}\right),
$$

avec $c$ le paramètre de forme (ou pente de Weibull) lié à la structure considérée, et $b$ le paramètre d'échelle lié à la valeur centrale de la distribution de probabilité de la variable $x=$ diamètre. Une valeur de $c<1$, distribution en «J renversé» est caractéristique des peuplements multispécifiques ou inéquiennes, tandis qu'une valeur $c>3,6$ est caractéristique des peuplements à prédominance d'individus âgés. Par ailleurs si $1<c<3,6$ cela désigne des peuplements avec prédominance d'individus jeunes ou de faible diamètre.

Une analyse log-linéaire, méthode itérative d'analyse de variance avec le logiciel SAS 9.1, est appliquée aux fréquences absolues observées et théoriques des différentes classes de diamètre afin de vérifier la qualité de l'ajustement de la distribution de Weibull à la distribution observée (Glèlè et Sinsin, 2009). 


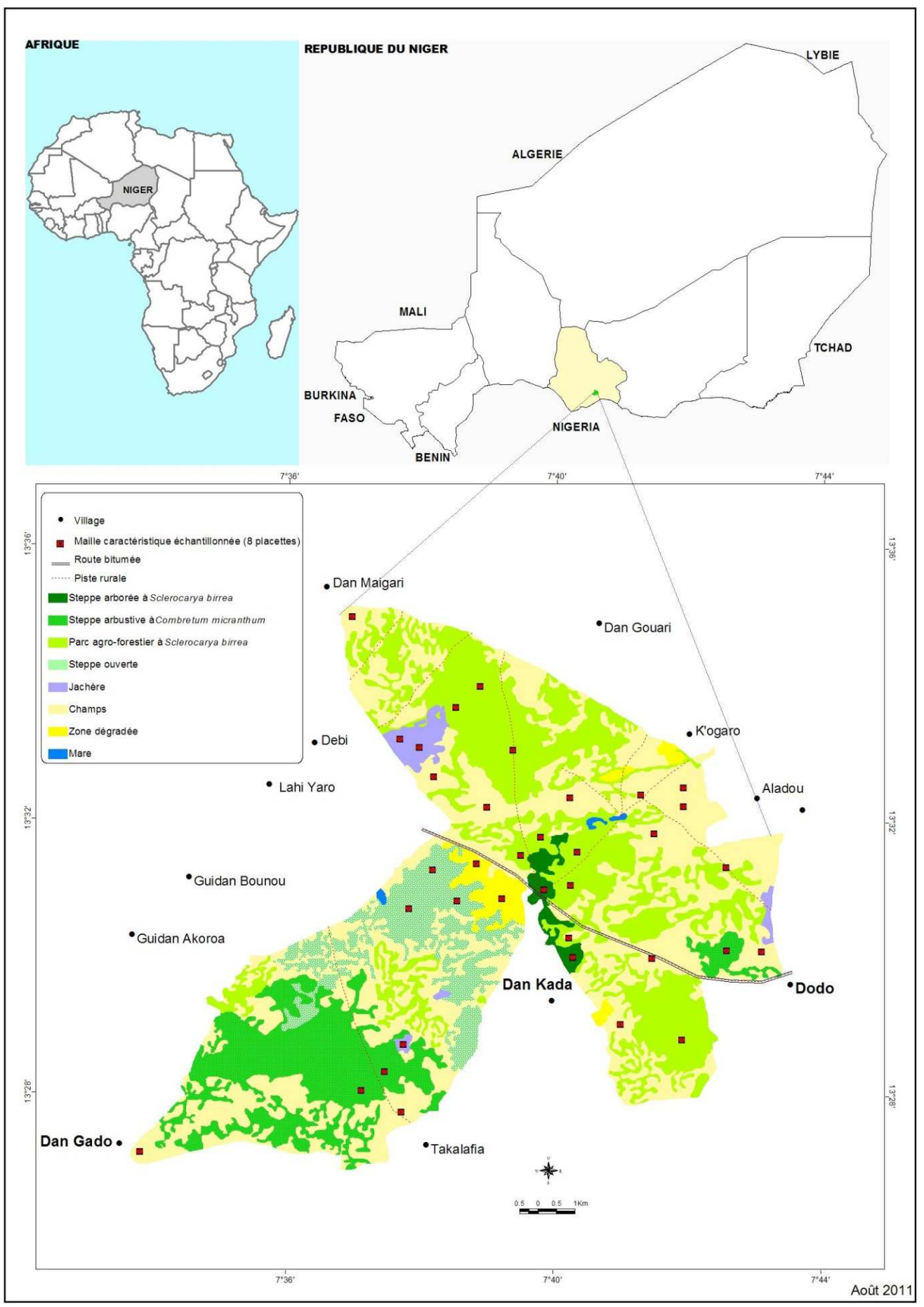

Figure 1 : Localisation, occupation des terres du complexe des forêts classées Dan kada Dodo Dan Gado et mailles caractéristiques échantillonnées. 
RESULTATS

\section{Composition floristique et diversité du peuplement ligneux \\ Richesse floristique}

L'inventaire floristique de la végétation ligneuse du complexe FDKDG a permis de dénombrer 31 espèces réparties en 23 genres et 15 familles. Les familles les plus dominantes (Figure 2) sont Mimosaceae (24,24\%), Combretaceae (15,15\%), Caesalpiniaceae et Capparaceae $(12,12 \%$ chacune), Anacardiaceae $(6,06 \%)$. Les dix autres familles représentent au total 30,3\% dont certaines ne sont représentées que par une seule espèce (Figure 2a).

La steppe arborée montre une dominance des Ceasalpinaceae et Mimosaceae (27,27\% chacune). Par contre, dans les unités des agrosystèmes constituées par les parcs agro-forestiers à Sclerocarya birrea, les champs, et les jachères, les familles les plus représentées sont les Combretaceae $(63,5 \%$; $61,07 \%$ et $72,3 \%$ respectivement), Capparaceae $(14,12 \% ; 7,51 \%$ et $10,52 \%$ respectivement) et enfin Mimosaceae (5,85\%; $12,95 \%$ et $10,92 \%$ respectivement). Sur le plan générique, les familles les mieux représentées sont Caesalpiniaceae (4 genres), Mimosaceae (3 genres) et enfin Anacardiaceae, Capparaceae et Combretaceae ( 2 genres). Toutes les autres familles ne sont représentées que par un seul genre. Parmi les genres, Acacia (6 espèces), Combretum (4 espèces) et Boscia (3 espèces) sont les mieux représentés.

Les espèces les plus fréquentes sont Guiera senegalensis (57,35\%), Boscia senegalensis (12,36\%); Combretum micranthum (7,08\%), Acacia senegal (5,76\%), Bauhina rufescens $(5,68 \%)$ et Sclerocarya birrea (3,13\%). Ce sont les espèces dominantes dans le complexe forestier car contribuant à plus de $89,95 \%$ au peuplement ligneux. Toutes les 25 autres espèces représentent 10,05\%. Dans les unités des agrosystèmes, les espèces les plus abondantes sont $G$. senegalensis $(54$ à $70 \%), B$. senegalensis (5 à 11\%), B. rufesecens (4 à $11 \%$ ) et A. senegal (5 à $9 \%$ ).

L'analyse de la composante ligneuse dans la steppe arborée (Figure 2b) montre la dominance des Ceasalpinaceae et Mimosaceae $(27,27 \%$ chacune). Cinq familles sont représentées de façon équilibrée $(9,09 \%)$ dans cette unité. $\mathrm{Ce}$ sont Anacardiaceae, Balanitaceae, Bomacaceae, Combretacea et Capparacea. Dans les unités des agrosystèmes constituées par les parcs à Sclerocarya birrea, les champs, et les jachères (Figures $2 \mathrm{f}$ et $2 \mathrm{~g}$ ), les familles les plus représentées sont Combretaceae (63,5\%; $61,07 \%$ et $72,3 \%$ respectivement), Mimosaceae $(5,85 \% ; 12,95 \%$ et $10,92 \%$ respectivement) et Capparaceae (14,12\%; $7,51 \%$ et $10,52 \%$ respectivement).

Le nombre de familles varie selon les unités d'occupation des terres. Au total, 15 familles ont été enregistrées, avec un nombre décroissant des unités des agrosystèmes (jachère, champs et parc à $S$. birrea) aux unités des formations naturelles (steppe arborée, steppe arbustive, steppe ouverte). Seulement quatre (4) espèces sont communes aux six principales unités d'occupation des terres. Il s'agit de Cassia sanguinea, $G$. senegalensis, Piliostignma reticulatum et $S$. birrea.

\section{Importance écologique des espèces}

Les espèces qui ont les plus fortes valeurs d'Indice de Valeur d'Importance (IVI) sont $G$. senegalensis $(114,70), \quad$ S. birrea (66,37), B. senegalensis $(25,21)$, A. senegal $(19,86)$, Combretum micranthum $(15,13)$ et $B$. rufescens $(14,57)$. Ces espèces marquent la physionomie de la végétation du complexe 
FDKDG. En effet, leur IVI représente $85,28 \%$ de l'IVI total (Tableau 1).

Types biologiques et affinités
phytogéographiques
Le spectre des types biologiques
(Figure 3) montre que la flore ligneuse du complexe forestier est dominée par les microphanerophytes (23 espèces, 74,19\% du spectre brut et $94,28 \%$ du spectre pondéré) suivis par les mésophanérophytes (7 espèces, $22,58 \%$ du spectre brute et 5,66\% du spectre pondéré). Les nanophanérophytes représentent (avec une seule espèce) 3,23\% des types biologiques.

Le spectre des types phytogéographiques (Figure 4) montre que la flore ligneuse est dominée par les espèces Soudaniennes avec $35,71 \%$ du spectre brut et $31,8 \%$ du spectre pondéré suivies des espèces Soudano-zambéziennes avec $25 \%$ du spectre brut et $59,5 \%$ du spectre pondéré. Les types phytogéographiques les moins représentés sont les espèces Plurirégionales africaines avec $14 \%$ du spectre brute et $1,55 \%$ du spectre pondéré.

\section{Indices de diversité spécifique}

Pour l'ensemble du complexe forestier, l'indice de diversité de Shannon $\left(\mathrm{H}^{\prime}\right)$ est de 2,34 bits et l'équitabilité de Pielou (E) de 0,47 bits (Tableau 2). A l'échelle des différentes unités d'occupation des terres, la richesse spécifique la plus élevée est observée au niveau des champs (25 espèces) suivis par les jachères (20 espèces) et représentent respectivement $80,6 \%$ et $64,5 \%$ du nombre total des espèces ligneuses recensées dans le complexe forestier.

\section{Caractéristiques dendrométriques}

La densité moyenne des ligneux dans le complexe FDKDG est de 425,19 $\pm 10,08$ individus/hectare. Les espèces les plus représentées sont G. senegalensis $(57,17 \%)$,
B. senegalensis $(12,83 \%), \quad$ C. micranthum $(6,90 \%)$, A. senegal $(5,70 \%)$, B. rufescens $(5,47 \%)$ et $S$. birrea $(3,15 \%)$. Les densités par unité d'occupation des terres varient entre $114,06 \pm 75,26$ individus/ha en zone dégradée à 809,37 $\pm 388,05$ individus/ha dans la steppe arbustive (Tableau 3).

La surface terrière est un critère permettant de juger de l'état d'une essence dans un peuplement. Pour l'ensemble du peuplement ligneux elle est de $0,76 \mathrm{~m}^{2} / \mathrm{ha}$. La surface terrière étant étroitement liée au diamètre, les unités d'occupation des terres renfermant beaucoup d'individus de petit diamètre présentent de faibles surfaces terrières. Ainsi, les surfaces terrières les plus importantes sont observées dans les steppes arborées $\left(3,21 \mathrm{~m}^{2} / \mathrm{ha}\right)$ suivie par les parcs agroforestier à $S$. birrea $\left(1,16 \mathrm{~m}^{2} / \mathrm{ha}\right)$. Les faibles valeurs s'observent dans la steppe arbustive $\left(0,14 \mathrm{~m}^{2} / \mathrm{ha}\right)$; les jachères $(0,37$ $\left.\mathrm{m}^{2} / \mathrm{ha}\right)$ et les champs $\left(0,58 \mathrm{~m}^{2} / \mathrm{ha}\right)$.

Du point de vue spécifique, la surface terrière varie suivant les espèces : $S$. birrea $\left(0,46 \mathrm{~m}^{2} / \mathrm{ha}\right) ;$ Balanites aegyptiaca $(0,04$ $\mathrm{m}^{2} / \mathrm{ha}$ ); le groupe des Acacia plantés ( $A$. senegal, A seyal, A. nilotica, A. laeta), et Faidherbia albida $\left(0,03 \mathrm{~m}^{2} /\right.$ ha chacun $)$. En termes de dominance relative (part de la surface terrière totale), S. birrea confirme sa prépondérance (Figure 5) en contribuant pour $60,11 \%$, suivi par le groupe des Acacias plantés $(16,91 \%)$.

Le recouvrement global du complexe FDKDG est de 5,55\%. L'unité d'occupation des terres la plus couverte est la steppe arborée avec 25,91\%. La dominance des arbustes dans la steppe arbustive se traduit par un faible recouvrement $2,16 \%$. On note un faible recouvrement des parcs agroforestiers à S. birrea $(3,88 \%)$ et des jachères $(4,47 \%)$ par rapport au recouvrement des champs $(7,96 \%)$. 


\section{Structure des ligneux}

La distribution des individus par classe de diamètre dans le complexe forestier est illustrée par la Figure 6. Les individus de petit diamètre représentent $61,43 \%$ (classes 1 et 2 cumulées) de la population totale (Figure 6a). Par contre, les individus constituant les classes de diamètre supérieur à $50 \mathrm{~cm}$ ne représentent que $0,82 \%$. L'analyse de la structure en diamètre des ligneux présente une allure en « $\mathrm{J}$ renversé » et s'ajuste à la distribution théorique de Weibull avec le paramètre de forme $\mathrm{c}=1,039(1<C<3,6)$, caractéristique des peuplements avec une prédominance des individus jeunes ou de petits diamètres.

La répartition par classes de diamètre des individus suivant les différentes unités d'occupation des terres étudiées montre une allure non gaussienne pour toutes les unités à l'exception des champs et présente une asymétrie droite $(1<\mathrm{c}<3,6)$, caractéristique des peuplements avec une prédominance relative des individus jeunes ou de faibles diamètres. Dans la steppe arborée, les individus de diamètre compris entre 20 et 25 $\mathrm{cm}$ sont les plus abondants soit $32,61 \%$; ceux de diamètre supérieur à $45 \mathrm{~cm}$ ne sont que de $2,17 \%$. Dans les unités de steppes arbustives, jachères et des parcs agroforestiers à $S$. birrea, les individus de diamètre compris entre 5 et $10 \mathrm{~cm}$ sont les plus abondants et représentent respectivement $40 \%, 57,14 \%$ et $35,08 \%$. La classe de diamètre supérieur à $40 \mathrm{~cm}$ est très faiblement représentée et ne se retrouve que dans les unités de la steppe arborée et des parcs agroforestiers à $S$. birrea .

La distribution des classes de diamètre observée dans les unités d'occupation des terres constituées par les champs s'ajuste avec une distribution théorique de Weibull avec un paramètre de forme $\mathrm{c}=0,9244(\mathrm{C}<1)$. Cette distribution est caractéristique des peuplements multispécifiques ou inéquiennes. A ce niveau, les individus de diamètre compris entre 5 et $10 \mathrm{~cm}$ sont les plus abondants à l'hectare et représentent $66,67 \%$ $\mathrm{du}$ peuplement tandis que les individus de diamètre supérieur à $35 \mathrm{~cm}$ y sont quasi absents $(0,22 \%)$.

Les résultats de l'analyse log-linéaire effectuée pour chacune des structures indiquent un bon ajustement des données à la distribution de Weibull pour les différentes unités d'occupation des terres $(p>0,05)$ au seuil de $5 \%$.

\section{Régénération}

La densité de régénération dans le complexe forestier est de 4333,34 tiges /hectare (Tableau 3). A l'échelle des différentes unités d'occupation des terres, la steppe arborée présente la plus faible régénération (495,32 tiges/ha) et les jachères présentent le grand effectif de régénération (8389,59 tiges/ha). Cependant, la dynamique se traduit par une chute significative du nombre de tiges entre la première classe $\mathrm{C} 1$ ( $\mathrm{h}<1,30 \mathrm{~m}$ ) montrant dans la quasi-totalité des unités, plus de $60 \%$ des jeunes tiges n'arrivent pas à s'affranchir de cette première classe pour passer à la classe suivante $\mathrm{C} 2(\mathrm{~h}>1,30)$. Du point de vue spécifique, les valeurs élevées de densité du semis naturel sont observées au niveau de $G$. senegalensis; $P$. reticulatum et $S$. birrea. En outre, certaines espèces à fort usage socio-économique comme S. birrea, Bombax costatum, B. aegyptiaca montrent une quasi absence des juvéniles. 
Tableau 1: Récapitulatif des paramètres dendrométriques des espèces dans le complexe des forêts classées de Dan kada Dodo-Dan Gado.

\begin{tabular}{|c|c|c|c|c|}
\hline Espèces & $\begin{array}{c}\text { Densité } \\
\text { relative } \\
(\%)\end{array}$ & $\begin{array}{c}\text { Surface } \\
\text { terrière } \\
\text { relative }(\%)\end{array}$ & $\begin{array}{c}\text { Fréquence } \\
\text { relative } \\
(\%)\end{array}$ & IVI \\
\hline Guiera senegalensis J.G. Gmel. & 57,17 & 0,18 & 57,35 & 114,7 \\
\hline Sclerocarya birrea (A. Rich.) Hochst. & 3,15 & 60,09 & 3,13 & 66,37 \\
\hline Boscia senegalensis (Pers.) Lam. Ex Poir. & 12,83 & 0,02 & 12,36 & 25,21 \\
\hline Acacia senegal (L.) Willd. & 5,7 & 8,39 & 5,76 & 19,86 \\
\hline Combretum micranthum G.Don. & 6,9 & 1,15 & 7,08 & 15,13 \\
\hline Bauhinia rufescens Lam. & 5,47 & 3,42 & 5,68 & 14,57 \\
\hline $\begin{array}{l}\text { Acacia nilotica (L.) Willd. ex. Del. subsp. } \\
\text { adstringens (Schum. et Thonn.) Roberty. }\end{array}$ & 1,06 & 7,53 & 0,94 & 9,53 \\
\hline Balanites aegyptiaca $($ L.) Del. & 0,95 & 4,87 & 0,96 & 6,78 \\
\hline Faidherbia albida (Del.) A. Chev. & 1,45 & 3,87 & 1,42 & 6,74 \\
\hline Piliostigma reticulatum (DC.) Hochst. & 1,41 & 2,47 & 1,36 & 5,23 \\
\hline Diospyros mespiliformis Hochst. ex. A. DC. & 0,02 & 4,5 & 0,02 & 4,54 \\
\hline Maerua crassifolia Forsk. & 1,47 & 0 & 1,42 & 2,89 \\
\hline Cassia singueana Del. & 0,97 & 0,09 & 0,92 & 1,98 \\
\hline Acacia radiana & 0,31 & 0,68 & 0,29 & 1,28 \\
\hline Commiphora africana (A.Rich.) Engl. & 0,02 & 0,96 & 0,02 & 1 \\
\hline Acacia seyal Del & 0,04 & 0,6 & 0,08 & 0,73 \\
\hline Stereospermum kunthianum Cham. & 0,17 & 0,19 & 0,17 & 0,52 \\
\hline Calotropis procera (Ait.) R. Br. & 0,21 & 0 & 0,21 & 0,42 \\
\hline Boscia angustifolia A. Rich. & 0,08 & 0 & 0,21 & 0,29 \\
\hline Ziziphus mauritiana Lam & 0,12 & 0 & 0,15 & 0,27 \\
\hline Acacia holocericea DC. & 0,08 & 0 & 0,08 & 0,17 \\
\hline Annona senegalensis Pers. & 0,08 & 0 & 0,06 & 0,15 \\
\hline Lannea microcarpa Engl. et K. Krauze & 0,06 & 0 & 0,06 & 0,12 \\
\hline Combretum glutinosum Perr ex DC & 0,04 & 0 & 0,04 & 0,08 \\
\hline Hyphaene thebaica $(\mathrm{L}$.$) Mart.$ & 0,04 & 0 & 0,04 & 0,08 \\
\hline Parkia biglobosa (Jacq.) Benth. & 0,04 & 0 & 0,04 & 0,08 \\
\hline Tamarindus indica $\mathrm{L}$ & 0,04 & 0 & 0,04 & 0,08 \\
\hline Bombax costatum Pellegr. & 0,04 & 0 & 0,02 & 0,06 \\
\hline Acacia laeta R.Br. Ex Benth. & 0,02 & 0 & 0,02 & 0,04 \\
\hline Azadirachta indica A. Juss. & 0,02 & 0 & 0,02 & 0,04 \\
\hline Combretum aculeatum Vent. & 0,02 & 0 & 0,02 & 0,04 \\
\hline Somme & 100 & 100 & 100 & 300 \\
\hline
\end{tabular}


Tableau 2: Synthèse des indices de diversité au sein des différentes unités d'occupation des terres dans le complexe des forêts classées de Dan kada Dodo-Dan Gado.

\begin{tabular}{|c|c|c|c|c|c|c|c|c|}
\hline \multirow[t]{2}{*}{ Indices } & \multirow{2}{*}{$\begin{array}{l}\text { Total } \\
\text { forêt }\end{array}$} & \multicolumn{7}{|c|}{ Unités d'occupation des terres } \\
\hline & & Steppe arborée & Steppe arbustive & Steppe ouverte & Zone dégradée & Jachères & Parc à Sclerocarya birrea & Champs \\
\hline Richesse spécifique (S) & 31 & 11 & 11 & 7 & 11 & 13 & 20 & 25 \\
\hline Indice de Shannon $\left(\mathrm{H}^{\prime}\right)$ & 2,34 & 2,34 & 1,85 & 0,50 & 2,76 & 1,78 & 2,30 & 2,38 \\
\hline Equitabilité de Pielou (E) & 0,47 & 0,68 & 0,53 & 0,18 & 0,80 & 0,48 & 0,53 & 0,51 \\
\hline Diversité maximale (H' max) & 4,95 & 3,46 & 3,46 & 2,81 & 3,46 & 3,7 & 4,32 & 4,64 \\
\hline
\end{tabular}

Tableau 3: Caractéristiques dendrométriques et de la régénération naturelle des ligneux dans les forêts de Dan Kada Dodo-Dan Gado par unité d'occupation des terres.

\begin{tabular}{|c|c|c|c|c|c|c|c|c|c|c|}
\hline \multirow[b]{2}{*}{$\begin{array}{l}\text { Unités d'occupation } \\
\text { des terres }\end{array}$} & \multirow{2}{*}{$\begin{array}{c}\text { Densité } \\
\text { (Nombre } \\
\text { d'individus/ha) }\end{array}$} & \multicolumn{4}{|c|}{ Densité de Régénération (Nombre de tiges/ha) } & \multicolumn{3}{|c|}{ Taux de régénération $(\%)$} & \multirow[b]{2}{*}{$\begin{array}{l}\text { Surface terrière } \\
\qquad\left(\mathbf{m}^{2} / \mathbf{h a}\right)\end{array}$} & \multirow[b]{2}{*}{$\begin{array}{c}\text { Recouvrement } \\
(\%)\end{array}$} \\
\hline & & $\begin{array}{c}\text { Hauteur } \\
<\mathbf{1 , 3 0 m}\end{array}$ & $\begin{array}{l}\text { Hauteur } \\
>1,30 \mathrm{~m}\end{array}$ & $\begin{array}{c}\text { Semis } \\
\text { naturel }\end{array}$ & Totale & $\begin{array}{c}\text { Hauteur } \\
<\mathbf{1 , 3 0 m}\end{array}$ & $\begin{array}{l}\text { Hauteur } \\
>1,30 \mathrm{~m}\end{array}$ & $\begin{array}{c}\text { Semis } \\
\text { naturel }\end{array}$ & & \\
\hline Global forêt & $425,19 \pm 410,85$ & 3848,44 & 417,19 & 67,71 & 4333,34 & 88,20 & 9,56 & 1,55 & 0,76 & 5,57 \\
\hline Steppe arborée & $170,31 \pm 87,66$ & 331,25 & 115,63 & 48,44 & 495,32 & 58,24 & 20,33 & 8,52 & 3,21 & 25,91 \\
\hline Zone dégradée & $114,06 \pm 75,26$ & 742,19 & 9,38 & 10,94 & 762,51 & 96,94 & 1,22 & 1,43 & - & - \\
\hline Steppe ouverte & $455,2 \pm 588,10$ & 3417,71 & 102,08 & 38,54 & 3558,33 & 95,99 & 2,87 & 1,08 & - & - \\
\hline Steppe arbustive & $809,37 \pm 388,05$ & 5626,04 & 2211,46 & 519,79 & 8357,29 & 67,24 & 26,43 & 6,21 & 0,14 & 2,16 \\
\hline Jachère & $782,29 \pm 415,49$ & 6356,25 & 2021,88 & 11,46 & 8389,59 & 75,49 & 24,01 & 0,14 & 0,37 & 4,47 \\
\hline $\begin{array}{l}\text { Parc agroforestier à } \\
\text { Sclerocarya birrea }\end{array}$ & $474,65 \pm 404,8$ & 5146,53 & 98,61 & 55,90 & 5301,04 & 96,44 & 1,85 & 1,05 & 1,16 & 3,88 \\
\hline Champs & $262,5 \pm 294,66$ & 3134,15 & 62,50 & 7,59 & 3204,24 & 96,77 & 1,93 & 0,23 & 0,58 & 7,96 \\
\hline
\end{tabular}



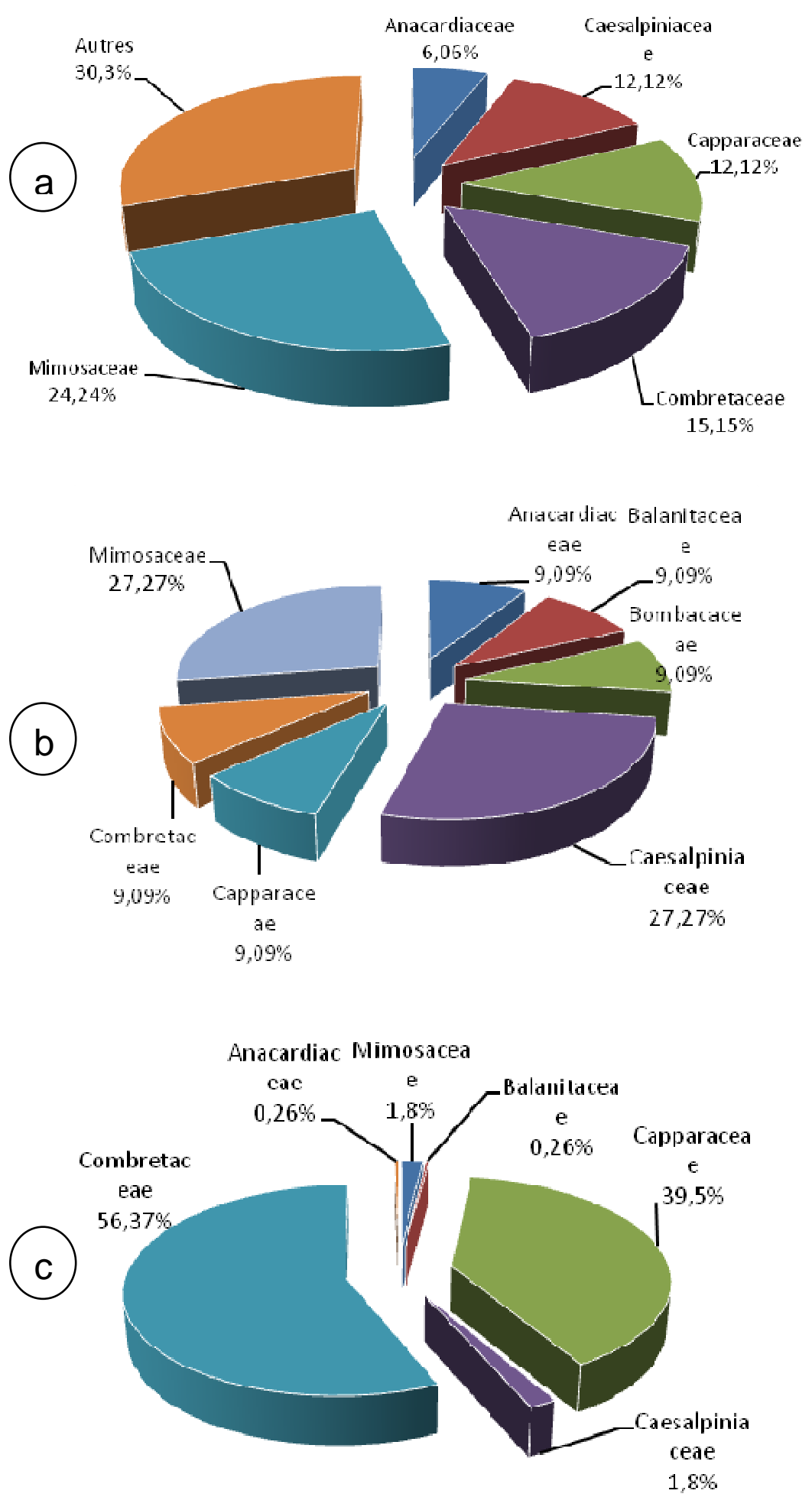

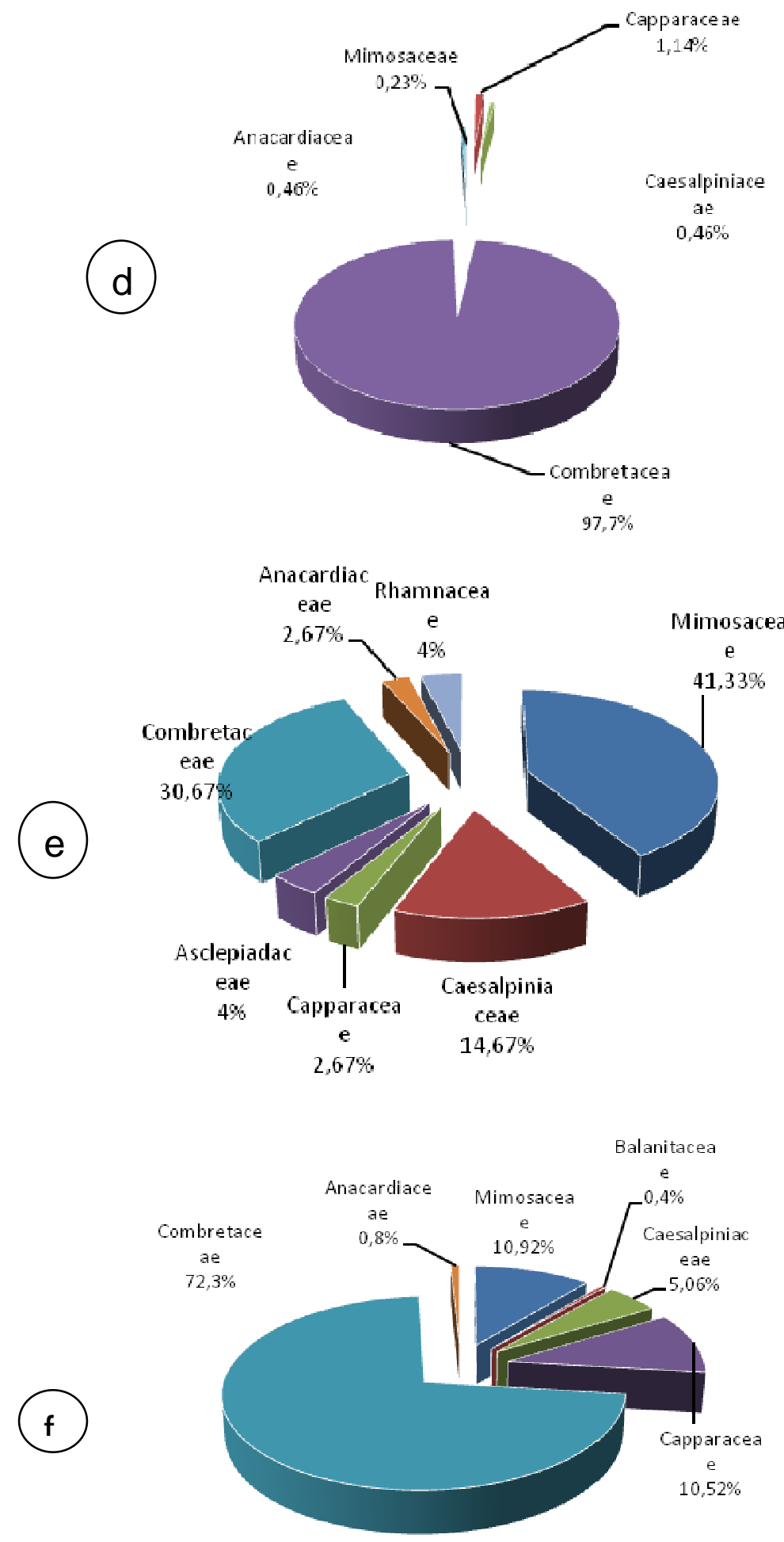

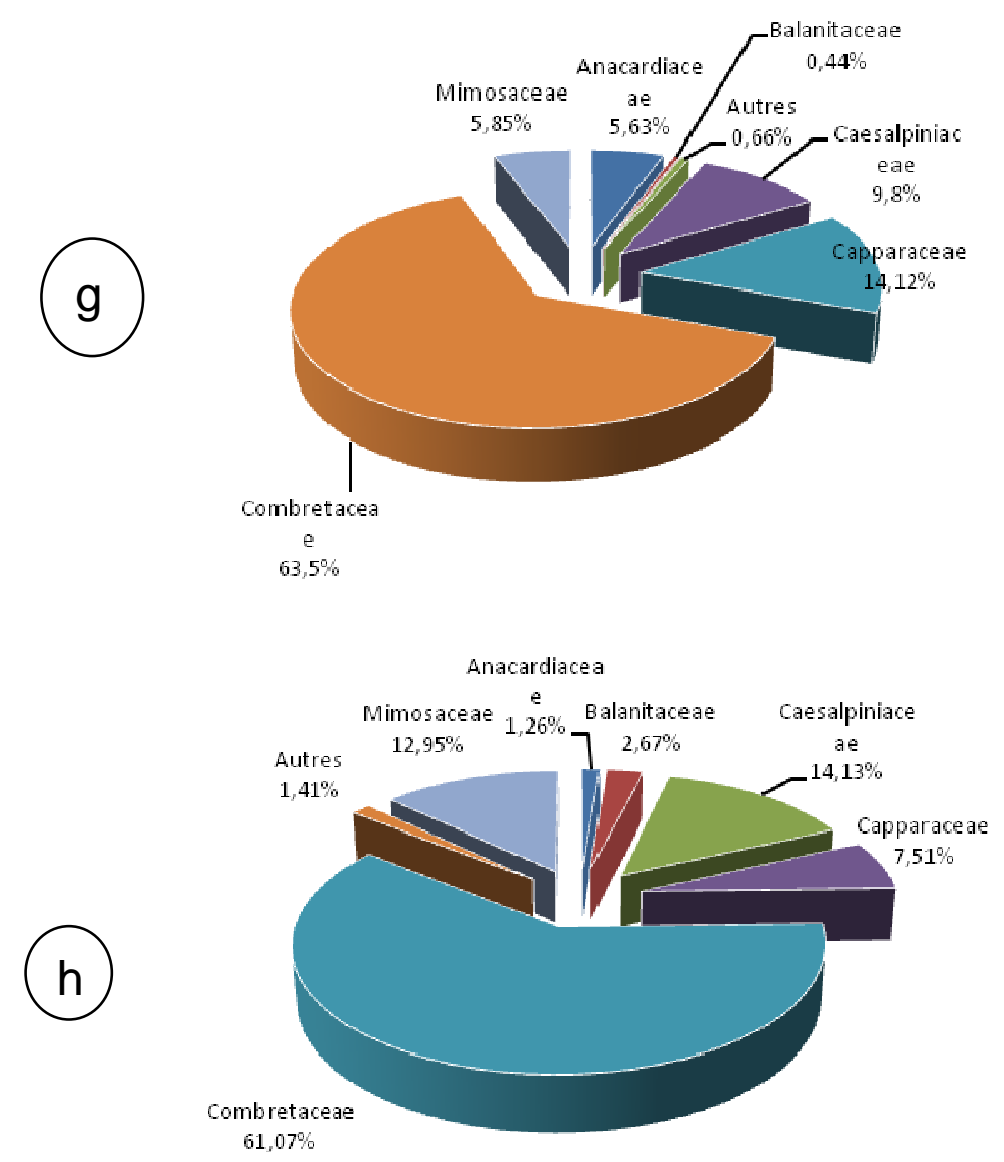

Figure 2: Spectre des familles des espèces ligneuses. $a=$ Total complexe Forêt, $b=$ Steppe arborée, $\mathrm{c}=$ Steppe arbustive, $\mathrm{d}=$ Steppe ouverte, e= Zone dégradée, $\mathrm{f}=$ Jachère, $\mathrm{g}=$ Parc à Sclerocarya birrea, $\mathrm{h}=$ Champs.

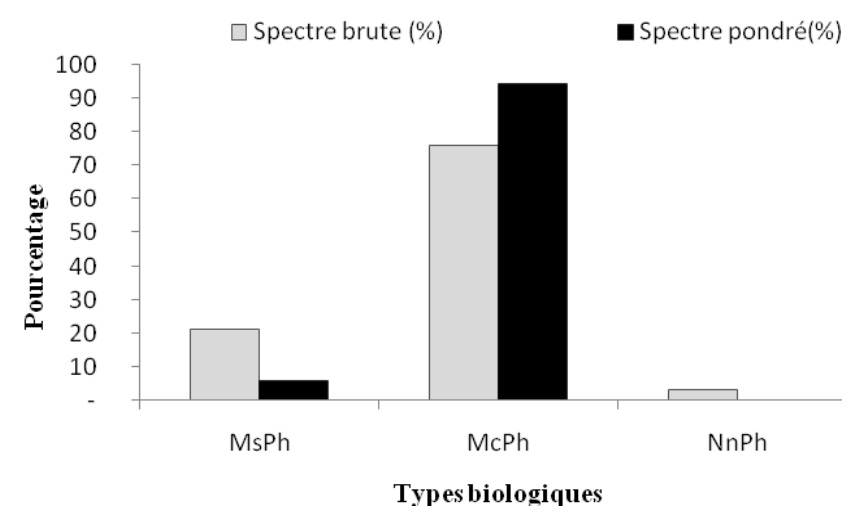

Figure 3: Spectre biologique des phanérophytes. $\mathrm{MsPh}$ : mésophanérophytes; $\mathrm{McPh}$ : microphanérophytes; $\mathrm{NnPh}$ : nanophanérophytes. 


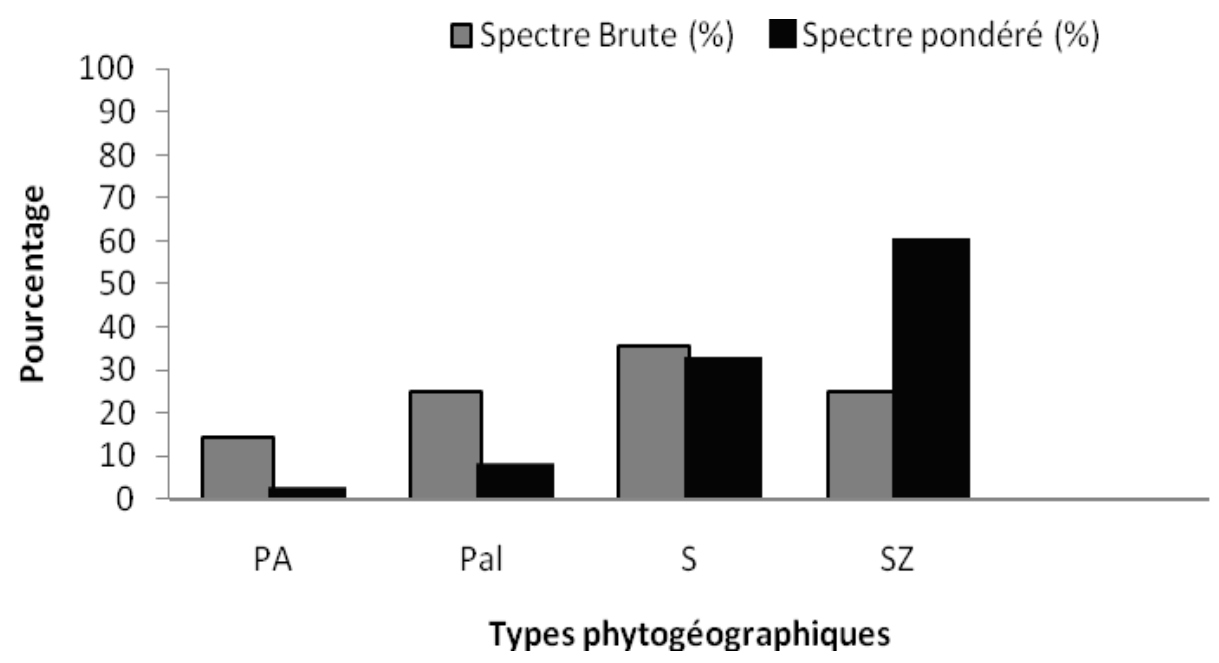

Figure 4 : Spectre phytogéographique. S: élément-base soudanien; SZ: espèces soudano-zambéziennes; PA: autres espèces pluri-régionales africaines; $\mathrm{Pal}$ : espèces paléotropicales.

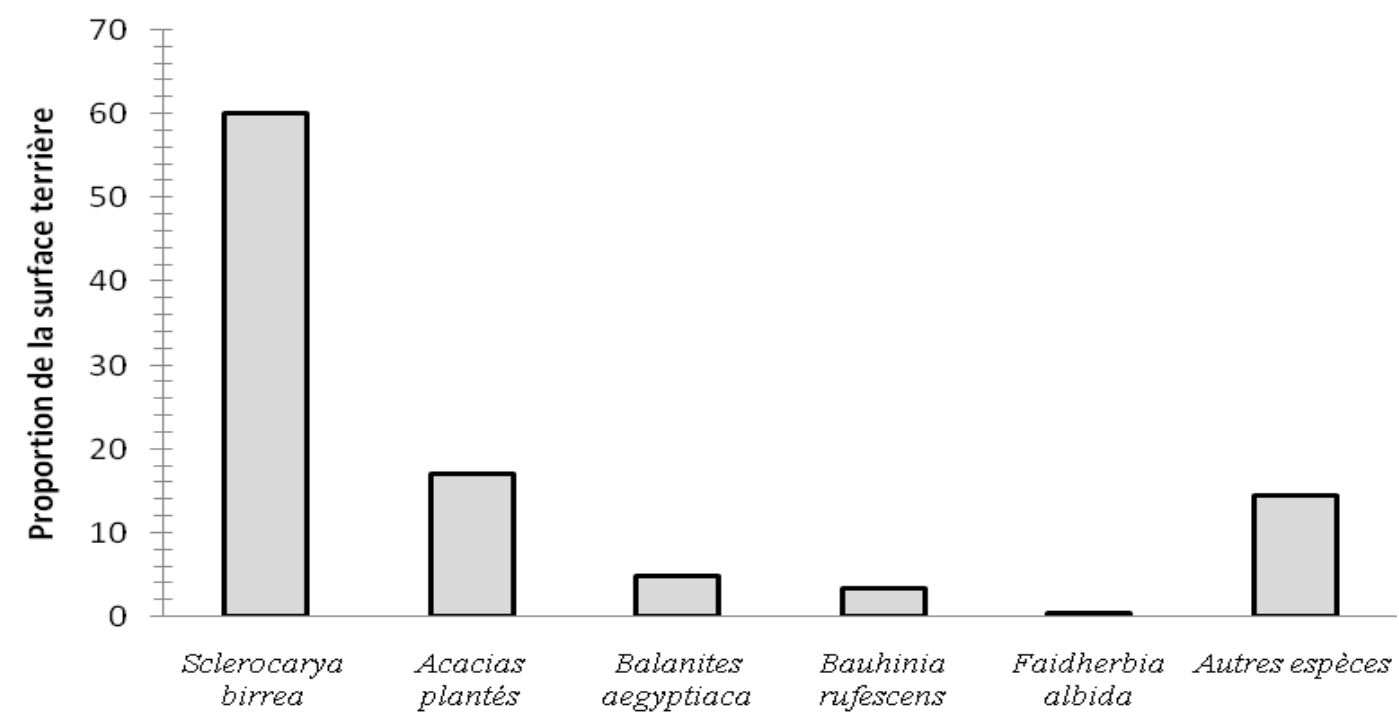

Espèces

Figure 5: Contribution relative des principales espèces et groupes d'espèces à la surface terrière. 

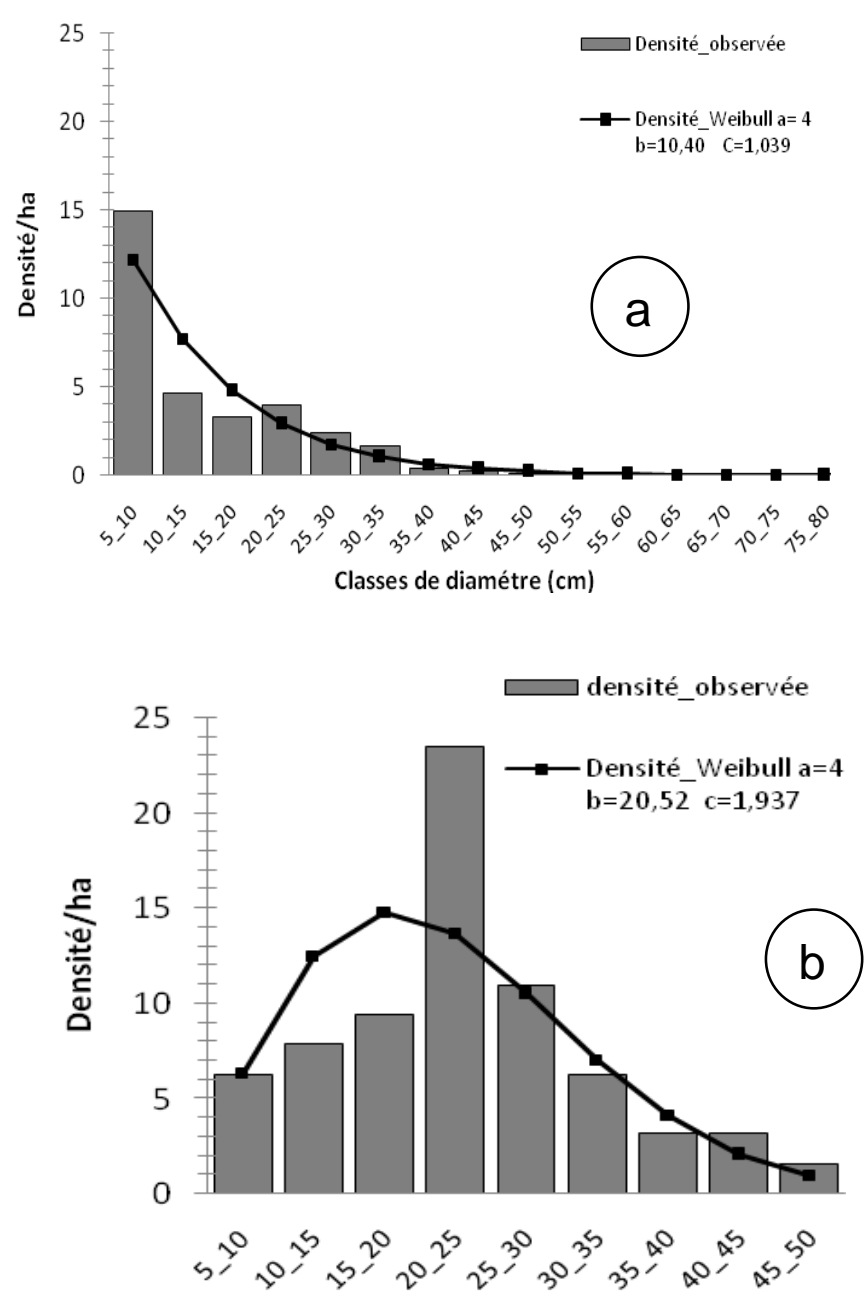

Classes de diamétre $(\mathrm{cm})$

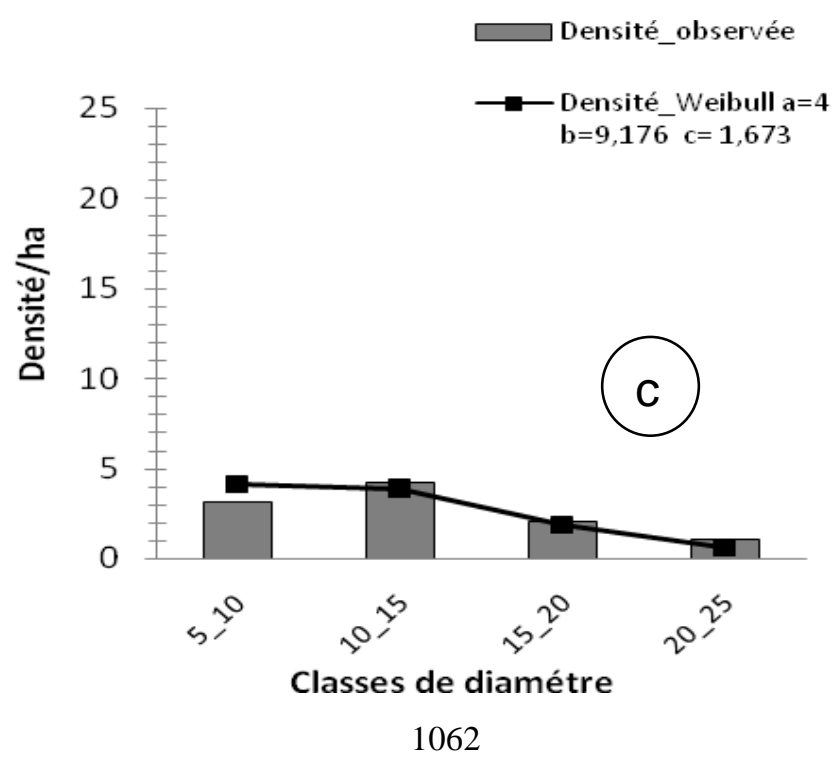




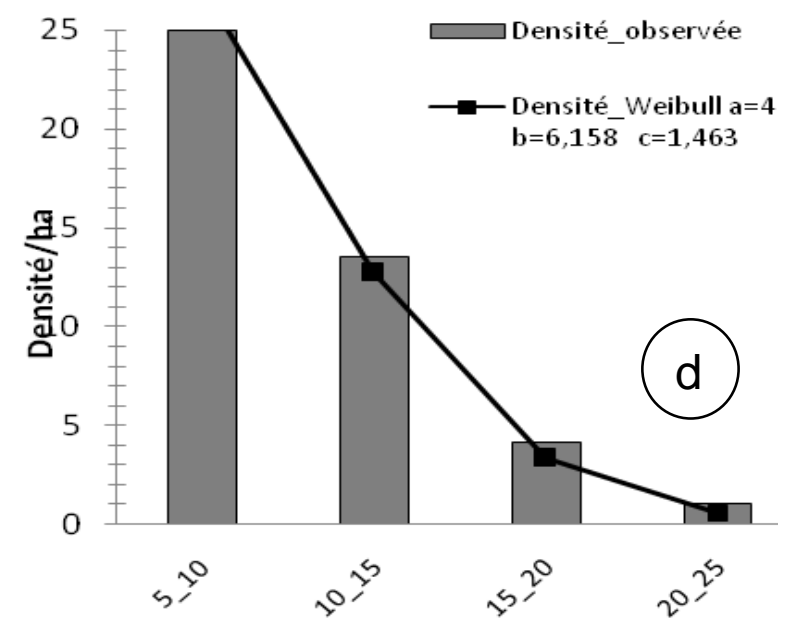

Classes de diamétre $(\mathrm{cm})$
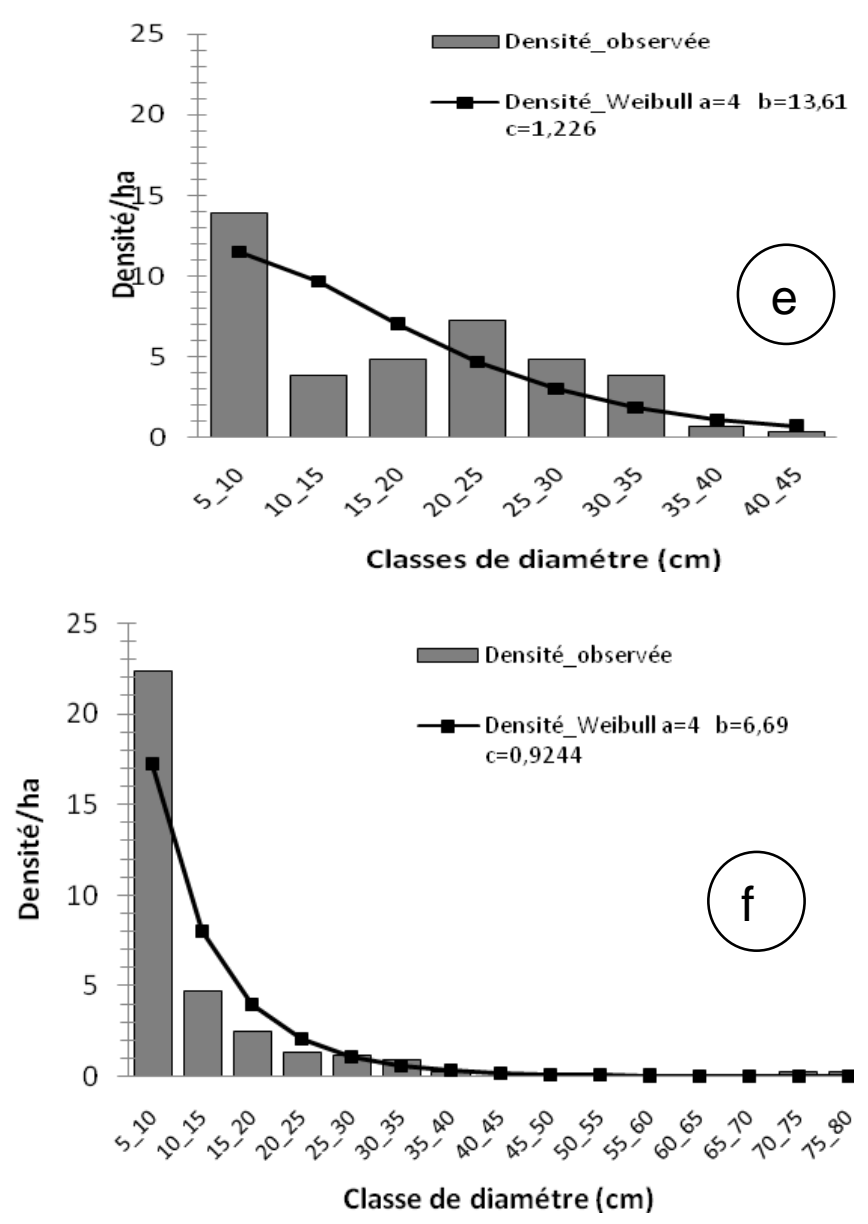

Figure 6: Structure démographique des essences ligneuses par classes de diamètre et par unité d'occupation des terres. a) complexe des forêts ; b) steppe arborée ; c) steppe arbustive; d) jachères; e) parc à Sclerocarya birrea; f) champs. 


\section{DISCUSSION \\ Composition floristique de la végétation ligneuse}

La richesse floristique du complexe des forêts classées de Dan Kada Dodo-Dan Gado est inférieure à celle obtenue par certains travaux effectués dans les aires protégées du Niger (Mahamane, 2005; Agbodjogbé, 2011) et au Burkina (Mbayngone et al., 2008). Cette différence serait due aux conditions climatiques plus favorables et l'effort de protection.

Les Combretaceae et les Mimosaceae indicatrices d'un climat généralement sec (Aubreville, 1950 cité par Mbayngone et al., 2008) représentent $39,39 \%$ des individus observés. Cette importance numérique pourrait en partie s'expliquer par le mode de dissémination des espèces appartenant à ces familles. Les Combretaceae sont en effet caractérisées par leurs fruits ailés facilement disséminés par le vent alors que les Leguminosae-Caesalpinioideae, généralement fourragères, aux semences zoochores sont disséminées par les herbivores qui les consomment (Ouédraogo, 2009). Les aspects de la dominance de la famille des Combretaceae qui sont observés peuvent être également attribués à leur faculté de régénération naturelle par les semis, par le drageonnement et/ou par rejets de souches (Bellefontaine, 2005). Cette dominance des Mimosaceae et Combretaceae est également relevée par Dimobé et al. (2012) dans la région septentrionale du nord Togo.

Le spectre des types biologiques fait ressortir une dominance des microphanérophytes. Ceci vient confirmer le type physionomique le plus répandu dans la zone d'étude, en l'occurrence les formations arbustives (Saadou, 1990; Mahamane et al., 2007). A l'échelle des unités d'occupation des terres, on note une prédominance des mésophanérophytes dans les champs. Cette situation pourrait s'expliquer par l'effet sélectif des activités anthropiques (pratiques agricoles et coupes de bois) favorisant certaines espèces pour imprimer une physionomie à la végétation. L'abondance de G. senegalensis, arbuste héliophile et indicateur de sol appauvri (Thiombiano, 1996), confirme que ce complexe forestier est graduellement mis en culture comme l'ont souligné Abdourhamane et al. (2012).

Les types phytogéographiques sont des bons indicateurs du dynamisme ou de la stabilité des communautés végétales (Sinsin, 1993). Dans la zone d'étude, les espèces à distribution Soudano-Zambéziennes (SZ) suivies des Soudaniennes sont dominantes. Ces résultats sont comparables à ceux de Mahamane et al. (2007) qui révèlent la dominance de ces types phytogéographiques dans tout l'Ouest du pays. Selon Sinsin (1993), la forte proportion des espèces à large distribution est un indice de perturbation et indique que la flore perd de sa spécificité.

\section{Diversité floristique de la végétation ligneuse \\ Les formations naturelles (steppe} arborée, steppe arbustive et steppe ouverte) ont une richesse faible (11 espèces) tandis que les formations des agrosystèmes constituées par les jachères, les parcs agroforestiers à $S$. birrea et les champs sont relativement plus riches (20 espèces). La valeur de l'indice de Shannon trouvée est comparable à celle de l'observatoire Maradi-Mayahi-Dakoro comportant des réserves de forêts (Mahamane et al., 2007). Elle reste cependant faible par rapport à celle obtenue dans la réserve de faune de Tamou contiguë au Parc National du W du Niger où les activités anthropiques sont contrôlées (Mahamane et al., 2007). L'indice de diversité de Shannon (H') le plus élevé (2,76 bits) et d'équitabilité plus grande $(0,8$ bits) sont obtenus dans les zones dégradées. Selon Legendre et Legendre (1998) cité par Mahamane (2005), l'indice de Shannon a des valeurs fortes pour des espèces avec des recouvrements de même importance et il prend des valeurs faibles lorsque quelques espèces ont de forts recouvrements. Les faibles valeurs de l'équitabilité observées traduisent la dominance des effectifs par quelques espèces qui sont $G$. senegalensis, $B$. senegalensis et $C$. micranthum avec une fréquence totale de $76,79 \%$. Elles peuvent 
aussi indiquer l'état de stress de l'écosystème (Floret et Pontanier, 1982).

\section{Caractéristiques dendrométriques}

Les caractéristiques dendrométriques du complexe FDKDG (densité de population et surface terrière respectivement égales à $425,19 \pm 410,85$ individus/ha et $0,76 \mathrm{~m}^{2} / \mathrm{ha}$ ) sont plus faibles que celles trouvées par Peltier et al. (2009) dans la forêt classée de Tientiergou au Niger avec 705 tiges/ha de densité et $2,13 \mathrm{~m}^{2} /$ ha de surface terrière, et par Paré (2008) dans deux forêts classées au Burkina avec des densités d'arbres variant entre $531 \pm 88$ et $796 \pm 90$ arbres/ha et des valeurs pour les surfaces terrières variant entre $6,55 \pm 1,1$ et $15,92 \pm 3,4 \mathrm{~m}^{2} /$ ha. La faible densité des peuplements ligneux dans le complexe FDKDG serait due d'une part aux conditions climatiques précaires et d'autre part à la pression croissante (défrichements agricoles, prélèvements anarchiques de bois) exercée sur les formations forestières par les populations riveraines. Cet état de fait a été relevé par Abdourhamane et al. (2012) qui ont montré une tendance à l'expansion des zones sous cultures avec un taux moyen annuel de $3,80 \%$ au détriment des formations naturelles $\mathrm{du}$ complexe forestier. Selon Sounon et al. (2007), la croissance démographique accompagnée de certains modes d'exploitation entraînent une pression sur les terres avec pour conséquence une perturbation des équilibres environnementaux.

\section{Structure et dynamique des peuplements ligneux}

La distribution par classe de diamètre est utilisée pour comprendre la dynamique des arbres et peut être utilisée pour évaluer l'impact de la pression anthropique sur la population des arbres (Cunningham, 2001). A l'échelle du complexe forestier, la répartition des individus en classes de diamètre a révélé une diminution progressive du nombre d'individus lorsque la classe de diamètre augmente. Selon Whitmore (1990), les densités élevées des classes de faible diamètre assurent l'avenir de la formation naturelle tandis que les faibles densités des classes de gros arbres résultent de la sélection naturelle et sont en fait les semenciers qui assurent la pérennité du peuplement. Une telle distribution, est typique des populations stables, susceptibles de se renouveler par la régénération naturelle (Mbayngone et al., 2008). Cependant, Sambou (2004) ayant fait le même constat dans des forêts classées au Sénégal, a conclu qu'une analyse globale de la structure de la végétation cache un processus de dégradation qui affecte les populations de certaines espèces à fort potentiel socioéconomique. En effet, les espèces appétées comme $S$. birrea et $B$. aegyptiaca sont régulièrement émondées par les éleveurs pour leur bétail en saison sèche. Cette pratique constitue une menace pour la conservation de la biodiversité ligneuse car ce sont généralement les semenciers nécessaires pour le renouvellement du peuplement qui sont ébranchés.

La densité globale de régénération est faible au sein du peuplement ligneux. Cette dynamique semble être hypothéquée par l'effet des conditions environnementales défavorables (sécheresse, pratiques agricoles, pâturage). En effet, seulement $11,025 \%$ de la régénération appartiennent à la classe des individus affranchis $(\mathrm{h}>1,30 \mathrm{~m})$. Selon Kozlowski (2002), la longueur de la saison sèche, les fluctuations climatiques et les fortes pressions anthropiques rendent la régénération naturelle aléatoire, plus particulièrement la reproduction par semis naturel qui représente $2,53 \%$ dans notre cas.

$\mathrm{Du}$ point de vue spécifique, l'abondance des populations de $G$. sengalensis, $\quad$. micranthum, et $B$. senegalensis, pourtant exploitées pour le bois de feu, tient au fait que ces espèces sont probablement moins affectées par la sécheresse comme l'ont souligné Thiombiano (1996) et Traoré (1997). Ces espèces sont également caractérisées par une reproduction asexuée (rejets de souche et drageons) généralement proportionnelle au taux d'anthropisation (Bellefontaine, 2005). Par ailleurs, la réduction significative de la densité de régénération de $S$. birrea pourrait être liée à la pression pastorale. En effet, les jeunes 
pousses de $S$. birrea constituent une source d'alimentation appréciable pour les petits ruminants compromettant ainsi le recrutement de cette espèce.

\section{Conclusion}

L'étude conduite dans le complexe des forêts classées de Dan Kada Dodo-Dan Gado a contribué à une meilleure connaissance de la composition floristique et de la structure des peuplements ligneux dans les différentes unités d'occupation des terres. Elle a permis de ressortir que les Mimosaceae et les Combretaceae sont dominantes. Les formations naturelles se révèlent floristiquement pauvres avec une forte contribution des Combretaceae tandis que les agrosystèmes montrent d'une part une diversité plus élevée et d'autre part une structure plus stable, à la faveur des plantations additionnelles dans le cadre des contrats de culture. L'abondance des microphanérophytes suivis des mésophanérophytes montre l'aspect arbustif des peuplements ligneux. L'évaluation de la diversité spécifique par l'indice de Shannon et l'équitabilité montre une certaine relation avec la perturbation du milieu. Malgré une densité des ligneux relativement faible, la flore ligneuse du complexe présente des espèces à fort usage socioéconomique prioritaire pour la revalorisation. Ces atouts militent en faveur $\mathrm{du}$ renforcement des stratégies d'aménagement et de gestion durable du complexe forestier.

\section{REMERCIEMENTS}

Les auteurs remercient tous ceux qui ont contribué à la réalisation de ce travail. Ces remerciements vont particulièrement à l'endroit des lecteurs anonymes du manuscrit ainsi qu'aux responsables du Service de l'Environnement de Aguié pour leur disponibilité et leur amabilité.

\section{REFERENCES}

Abdourhamane H, Morou B, Mahamane A, Saadou M, Issaka A. 2012. Caractérisation de la dynamique spatiotemporelle de l'occupation des terres dans le complexe des forêts classées de Dan kada Dodo-Dan Gado (région de Maradi, Niger). Journal des Sciences de l'Environnement, 1(1): 16-26.

Agbodjogbé JG. 2011. Analyse de la Structure des Galeries Forestières de la Réserve Totale de Faune de Tamou (RTFT) en République du Niger. Mémoire de Master en biodiversité végétale tropicale, Université de paris, 50p.

Bellefontaine R. 2005. Pour de nombreux ligneux, la reproduction sexuée n'est pas la seule voie: analyse de 875 cas. Sécheresse, 16(4): 315-317.

Boulain N. 2004. Effet des facteurs climatiques et anthropiques dans l'évolution récente des écosystèmes tropicaux : modélisation spatialisée du bilan hydrique d'un petit bassin versant sahélien. Thèse de doctorat, Université Pierre et Marie Curie, Paris VI, 171p.

Breman H, Kessler JJ. 1995. Woody Plants in Agro-ecosystems of Semi-arid Regions, with an Emphasis on the Sahelian Countries. Springer Verlag: Berlin; $340 \mathrm{p}$.

Cunningham AB. 2001. Applied Ethnobotany. People Wild Plant Use and Conservation. People and Plants Conservation. Earth scan Publications Ltd: London; 300p.

Dimobé K, Wala K, Batawila K, Dourma M, Woegan YA, Akpagana K. 2012. Analyse spatiale des différentes formes de pressions anthropiques dans la réserve de faune de l'Oti-Mandouri (Togo). VertigO, la revue Electronique en Sciences de l'Environnement (En ligne), Hors-série $14 \mid$ septembre 2012, http://vertigo.revues.org/12423 ; DOI : 10.4000/vertigo. 12423

FAO. 2010. Evaluation des ressources forestières mondiales 2010. Rapport principal. Etude FAO, Forêts $\mathrm{N}^{\circ} 163$, Rome, Italie, 348p.

Floret C, Pontanier R. 1984. Aridité climatique, aridité édaphique. Bulletin de la Société Botanique de France, 131: 265-275. 
Ganaba S. 2008. Caractérisation, utilisations, tests de restauration et gestion de la végétation ligneuse au Sahel, Burkina Faso. Thèse de Doctorat d'Etat ès Sciences Naturelles, Université Cheikh Anta Diop, 287 p.

Glèlè Kakaï R, Sinsin B. 2009. Structural description of two Isoberlinia dominated vegetation types in the Wari-Maro Forest Reserve (Benin). South African Journal of Botany, 75: 43-51.

Gonzalez P, Tucker CJ, Sy H. 2012. Tree density and species decline in the African Sahel attributable to climate. Journal of Arid Environments, 78: 5564.

Guinko. S., 1984. Végétation de la HauteVolta. Thèse, Université de Bordeaux III, 2 tomes, 394p.

Hountondji YC. 2008. Dynamique environnementale en zones sahélienne et soudanienne de l'Afrique de l'Ouest : Analyse des modifications et évaluation de la dégradation du couvert végétal. Thèse de Doctorat en Sciences de l'Environnement, Université de LiègeBelgique, $153 \mathrm{p}$.

Kozlowski TT. 2002. Physiological ecological of natural regeneration of harvested and disturbed forest stands: implications for forest management. Forest Ecology and Management, 158: 195-221.

Larwanou M, Saadou M. 2005. Biodiversity of ligneous species in semi-arid to arid zones of south-western Niger according to anthropogenic and natural factors. Agriculture, Ecosystems and Environment, 105: 267-271.

Lebrun J-P, Stork AL. 1997. Enumération des Plantes à Fleurs d'Afrique Tropicale (Vol. IV). Conservatoire et Jardin botanique de Genève.

Mahamane A. 2005. Etudes floristique, phytosociologique et phytogéographique de la végétation du Parc Régional du W du Niger. Thèse de doctorat, Université Libre de Bruxelles, Laboratoire de Botanique systématique et de Phytosociologie, 484 p.
Mahamane A, Saadou M, Bakasso Y, Issaka A, Ichaou A, Saley K. 2007. Analyse diachronique de l'occupation des terres et caractéristiques de la végétation dans la commune de Gabi (région de Maradi, Niger). Sécheresse, 18(4): 296-304.

Mahamane A, Saadou M. 2008. Méthode d'étude de la flore et de la végétation tropicale. Project SUN-EU. Actes de l'atelier sur l'harmonisation des méthodes, $83 \mathrm{p}$.

Mbayngone E, Thiombiano A, Hahn-Hadjali K, Guinko S. 2008. Structure des ligneux des formations végétales de la Réserve de Pama (Sud-Est du Burkina Faso, Afrique de l'Ouest). Flora et Vegetatio Sudano-Sambesica, 11: 25-34.

Matsallabi A. 2008. Ressources ligneuses et problématique d'aménagement dans la forêt classée de Dan Kada-Dodo (Département d'Aguié). Rapport de stage pour l'obtention du diplôme de Maîtrise ès-sciences Agronomiques, Université Abdou Moumouni de Niamey, 61p.

Morou B. 2010. Impacts de l'occupation des sols sur l'habitat de la girafe au Niger et enjeux pour la sauvegarde du dernier troupeau de girafes de l'Afrique de l'Ouest. Thèse de doctorat, Université Abdou Moumouni de Niamey, 231p.

N'da HD, Adou CY, N'guessan EK, Koné M, Sagné YC. 2008. Analyse de la diversité floristique du parc national de la Marahoué, Centre-Ouest de la Côte d'Ivoire. Afrique Science, 04(3): 552-579

Oszwald J. 2005. Dynamique des formations agroforestières en Côte d'Ivoire (des années 1980 aux années 2000): Suivi par télédétection et développement d'une approche cartographique. Thèse de doctorat de Géographie, Université des Sciences et Technologies de Lille, 304p.

Ouédraogo O. 2009. Phytosociologie, dynamique et productivité de la végétation du parc national d'Arly (SudEst du Burkina Faso). Thèse de doctorat de l'Université de Ouagadougou, 188p.

Ozer A. Ozer P. 2005. Désertification au Sahel: crise climatique ou anthropique? Bulletin des Séances de l'Académie 
Royale des Sciences d'Outre-Mer, 51: 395-423.

Paré S. 2008. Land use dynamics, tree diversity and local perception of forest decline in southern Burkina Faso, West Africa. Doctoral Thesis, Swedish University of Agricultural Sciences, Faculty of Forest Sciences, Department of Forest Genetics and Plant Physiology, $78 \mathrm{p}$.

Peltier RJ, Dessard H, Gado Alzouma R, Ichaou A. 2009. Bilan après quinze ans de gestion communautaire d'une forêt villageoise de l'Ouest nigérien. Sécheresse, 20(4): 383-387.

Saadou M. 1990. La végétation des milieux drainés nigériens à l'Est du fleuve Niger. Thèse de doctorat, Université Niamey, Niger, 393p.

Sambou B. 2004. Evaluation de l'état, de la dynamique et des tendances évolutives de la flore et de la végétation ligneuses dans les domaines soudanien et subguinéen au Sénégal. Thèse de doctorat, Université Cheikh Anta Diop, 248p.

Sinsin B. 1993. Phytosociologie, écologie, valeur pastorale, production et capacité de charge des pâturages naturels du périmètre Nikki-Kalalé au Nord-Bénin. Thèse présentée en vue de l'obtention du grade de Docteur en Sciences Agronomiques. Université Libre de Bruxelles, $390 \mathrm{p}$.

Sounon BB, Sinsin B, Goura SB. 2007. Effets de la dynamique d'occupation du sol sur la structure et la diversité floristique des forêts claires et savanes au Bénin. Tropicultura, 25(4): 221-227.

Thiombiano A. 1996. Contribution à l'étude des Combretaceae dans les formations végétales de la région Est du Burkina Faso. Thèse de doctorat de $3^{\text {ème }}$ cycle, université de Ouagadougou, 220 p.

Traoré SA. 1997. Analyse de la flore ligneuse et de la végétation de la zone de Simenti (parc National du Niokolo Koba), Sénégal Oriental. Thèse de Doctorat de $3^{\text {ème }}$ cycle en biologie végétale, Université Cheikh Anta Diop de Dakar, $147 \mathrm{p}$.

Vroh Bi Tra A, Adou Yao CY, Kouamé D, N'da Dibi H, N'guessan KE. 2010. Diversités floristique et structurale sur le site d'une réserve naturelle volontaire à Azaguié, Sud-Est de la Côte d'Ivoire. European Journal of Scientific Research, 45(3): 411-421.

Whitmore TC. 1990. An Introduction to Tropical Rain Forest. Clarendo Press: Oxford; 225p. 